\title{
Touch the sky with your hands: a special Planetarium for blind, deaf, and motor disabled
}

\author{
Beatriz García, Javier Maya, Alexis Mancilla, Silvina Pérez Álvarez, \\ Mariela Videla, Diana Yelós and Angel Cancio \\ Instituto de Tecnologías en Deteccíon y Astropartículas Mendoza \\ Mendoza, Argentina \\ email: beatriz.garcia@iteda.cnea.gov.ar
}

The Planetarium for the blind, deaf, and motor disabled is part of the program on Astronomy and Inclusion of the Argentina Pierre Auger Foundation (FOPAA) and the Institute in Technologies and Detection of Astroparticles-Mendoza (ITeDAM).

The base of the proposal is the multi-sensorial perception of nature, with the approach to the starry sky from a new perspective: the Planetarium, where the stars are represented by LEDs and the hemisphere representing the sky is overhead, the implementation of new resources and tools for scientific outreach in general (like Geiger counters) and Astronomy in particular (Solar System models), designed for people with disabilities. The installation is aimed not only to the blind and visually impaired, but also the deaf and people with motor impediments. This space allows an approach to Astronomy, their concepts and discoveries, developing a sense of wonder before the comprehension of the natural world. The perception of a palpable and sensitive space by generating a particular environment, aims to bring the visitor to the conditions of the external natural environment at night. For these reasons, specific objectives are providing people with special educational needs, visually impaired and / or motor disabilities, a participatory learning and accessible resource, at the same time interesting and training, taking into account the accurately and strictly scientific knowledge, but also ensuring a playful interaction framework. This facility is installed at an appropriate area in Tecnópolis, the mega exhibition of Science and Technology, supported by the Ministry of Science, Technology and Productive Innovation of Argentina, with about 5000 visitors a day. (See Figure 1).

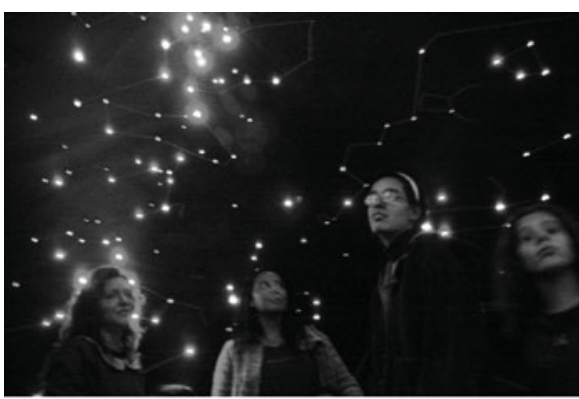

(a)

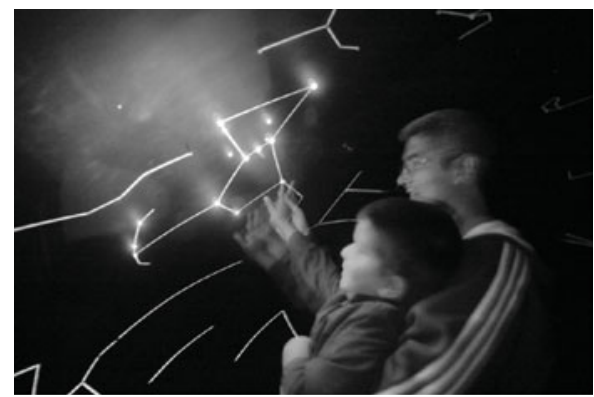

(b)

Figure 1. Touch the sky with your hands: a Planetarium for everyone 